\title{
Diversidad de las hidromedusas (Cnidaria) de la región nerítica del sureste de Tamaulipas, México
}

\author{
Martha Angélica Gutiérrez-Aguirre \\ Víctor Hugo Delgado-Blas \\ Adrián Cervantes-Martínez \\ Departamento de Ciencias y Humanidades \\ Universidad de Quintana Roo-Unidad Académica Cozumel
}

\section{Resumen}

Se analizan a escala estacional algunos indicadores de la estructura de la comunidad de hidromedusas que habitan en la región nerítica del sureste de Tamaulipas, México. Se realizaron colectas de noviembre de 1990 a octubre de 1991 en cinco estaciones por medio de arrastres superficiales circulares; se registraron 22 especies, pertenecientes a 19 géneros, 14 familias y cinco subclases. El meroplancton fue el de mayor riqueza específica (75 \%) y mayor abundancia (63.6\%), y el holoplancton presentó un menor número de especies y fue menos abundante (33.5\%). Las especies de mayor densidad fueron $\mathrm{Li}$ riope tetraphylla (Chamisso y Eysenhardt, 1821), Obelia sp. y Persa incolorata (McCrady, 1857). La máxima densidad de hidromedusas se observó a finales de la temporada de secas, mientras que los mayores índices de equidad y diversidad se presentaron en la temporada de lluvias, cuando las especies dominantes prácticamente se ausentaron. Las especies Coryne eximia (Allman, 1859), Slabberia halterata McCrady, 1859, Ectopleura dumortierii (Van Beneden, 1844) y E. ventricularis McCrady, 1859 constituyen nuevos registros para el norte del golfo de México. Modelos cuantitativos estimaron que la riqueza observada representó de 78.5 a $100 \%$ de la estimada; por último, el índice de diversidad de Shannon se relacionó significativamente con la temperatura del agua mínima $(\mathrm{rS}=0.8 ; \mathrm{p}<0.05)$ y máxima $(\mathrm{rS}=0.67 ; \mathrm{p}<0.05)$

\section{Palabras clave}

Abundancia, diversidad, golfo de México, riqueza, zooplancton marino.

Recibido: 13/04/2015 A Aceptado: 18/05/2015

* Correo electrónico: margutierrez@uqroo.edu.mx 


\title{
Diversity of Hydromedusae (Cnidaria) of the neritic region of southeastern Tamaulipas, Mexico
}

\author{
Martha Angélica Gutiérrez-Aguirre \\ Víctor Hugo Delgado-Blas \\ Adrián Cervantes-Martínez \\ Departamento de Ciencias y Humanidades \\ Universidad de Quintana Roo-Unidad Académica Cozumel
}

\begin{abstract}
Various indicators of the structure of Hydromedusae communities in the neritic region of southeastern Tamaulipas, México are anayzed in a seasonal scale. Bimonthly collections were carried out between november 1990 and october 1991 in five sites using superficial circular trawls; 22 species were registered belonging to 19 genuses, 14 families and five subclasses. Meroplankton represented the largest specific wealth (75 \%) and abundance (63.6 \%) while holoplankton presented a smaller number of species and was less abundant (33.5 \%). The species with higher density were Liriope tetraphylla (Chamisso y Eysenhardt, 1821), Obelia sp. and Persa incolorata (McCrady, 1857). The largest density of Hydromedusae was observed at the end of the dry season while the larger indices of equity and diversity were found in the rainy season when dominant species where practically absent. The species Coryne eximia (Allman, 1859), Slabberia halterata (McCrady, 1859), Ectopleura dumortierii (Van Beneden, 1844) y E. ventricularis (McCrady, 1859) constitute new records for the north of the Gulf of Mexico. Through quantitatve models it was estimated that the observed wealth represented 78.5 to $100 \%$ of the estimated one; lastly, the Shannon indice of diversity was significantly related to minimal ( $\mathrm{rS}=0.8 ; \mathrm{p}<0.05$ ) and maximal ( $\mathrm{rS}=0.67 ; \mathrm{p}<0.05$ ) water temperature.
\end{abstract}

\section{KEY WORDS}

Abundance, diversity, Gulf of Mexico, wealth, marine zooplankton. 


\section{Introducción}

Las medusas tienen una extraordinaria capacidad como depredadoras en el ambiente pelágico (Suárez-Morales, Segura-Puertas y Gasca, 1995: 243), asimismo pueden competir por el alimento con carnívoros y devorar organismos del zooplancton como copépodos, quetognatos, larvas de peces y aun otras medusas. Su capacidad de depredación está asociada con el tamaño que tengan, de ahí su amplio espectro trófico en el ecosistema marino, que además incluye animales de importancia comercial como peces, crustáceos y moluscos (Segura-Puertas, Celis y Chiaverano, 2009: 369). La función de las medusas en el ambiente marino ha sido destacada entre otras cosas por la particularidad de algunas especies que han sido designadas como indicadores biológicos de masas de agua (Raskoff, 2001: 121).

A pesar de su relevancia ecológica, se sabe poco de los componentes estructurales básicos de las medusas que habitan en las regiones costeras del norte del golfo de México (Segura-Puertas, Suárez-Morales y Celis, 2003: 2).

Con el objetivo de conocer la composición cualitativa y la abundancia relativa de las hidromedusas, así como de estimar las posibles variaciones de las poblaciones a lo largo del año, se estableció un programa de muestreos considerando aspectos hidrológicos y de zooplancton en la región costera sureste de Tamaulipas. Se han publicado pocos trabajos sobre este grupo zoológico en el golfo de México, entre ellos destacan los de Sears (1954: 273), Alvariño (1972), Phillips (1972), Correia-Valencia (1992), Canudas (1979), Signoret (1969) y Gómez-Aguirre (1991), realizados en lagunas costeras de la región.

Así, este estudio contribuye al conocimiento de la estructura comunitaria de medusas que habitan una región costera al sureste de Tamaulipas, en el área de influencia de la región marina prioritaria Laguna San Andrés (Arriaga-Cabrera et al., 1998). Con los datos obtenidos se ofrece una predicción de la riqueza específica del área.

\section{Metodología}

Se realizaron muestreos de noviembre de 1990 a octubre de 1991 en cinco estaciones distribuidas perpendicularmente en la línea de costa sureste del estado de Tamaulipas, México (figura 1) y durante tres temporadas climáticas de la 


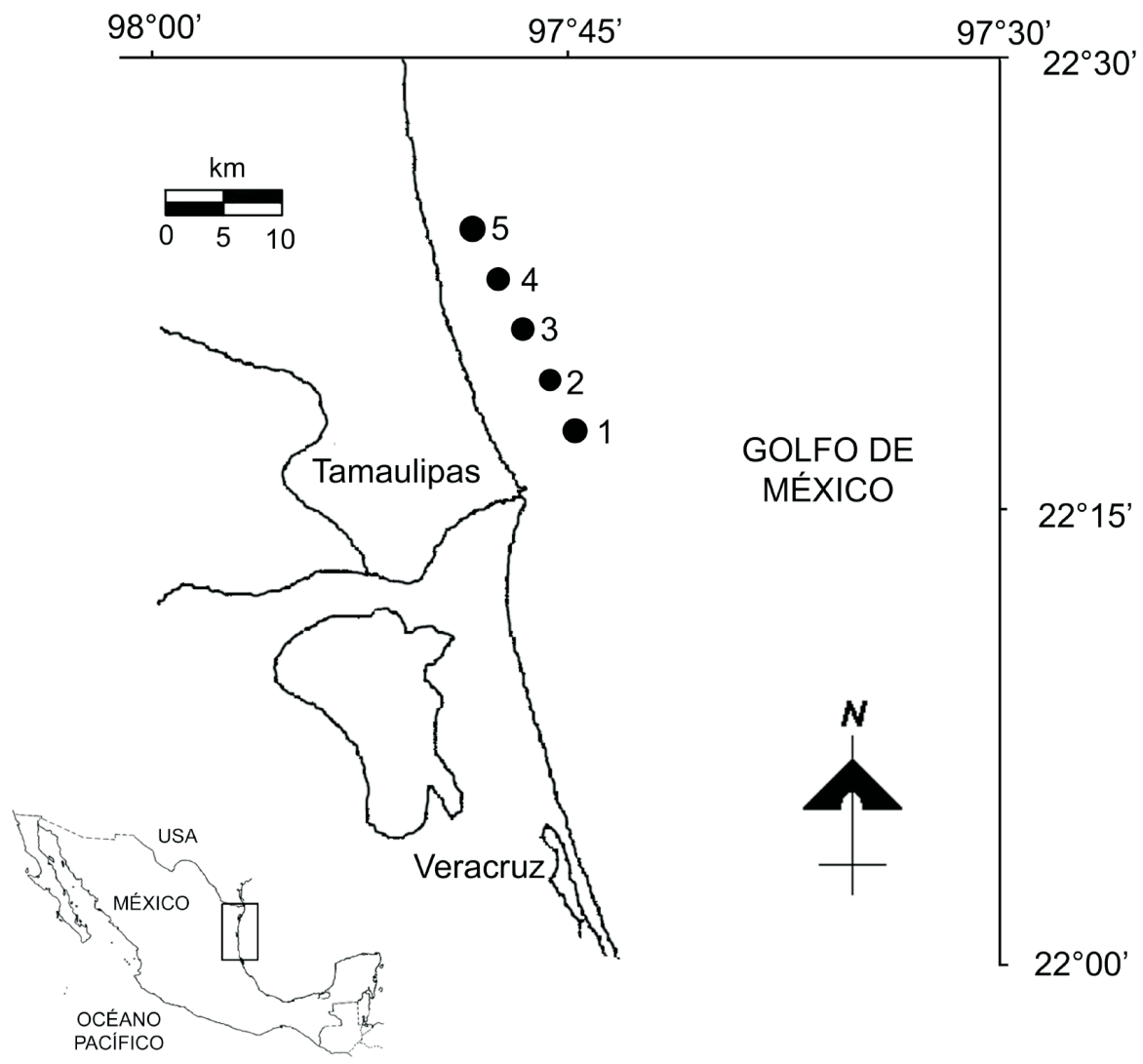

Figura 1.- Área de estudio y sitios de colecta (1-5).

región, establecidas como nortes (de noviembre a marzo), secas (de abril a julio) y lluvias (de agosto a octubre) (Ocaña-Luna, Hernández-Batún y Sánchez Ramírez, 2008: 200). El área analizada (playa Miramar) es una playa arenosa de pendiente ligera y de $6 \mathrm{~km}$ de longitud. Se encuentra limitada al sur por la escollera norte del río Pánuco. Se llevaron a cabo arrastres circulares superficiales de cinco minutos a dos nudos de velocidad; con una red cónica de $50 \mathrm{~cm}$ de diámetro y $2 \mathrm{~m}$ de largo, con apertura de malla de $315 \mu \mathrm{m}$. El volumen de agua filtrada se determinó a través de un flujómetro General Oceanics Inc 2030R. En cada estación de muestreo se hicieron mediciones de temperatura con ayuda 
de un termómetro de mercurio de rango $0+375^{\circ} \mathrm{C}$ con precisión \pm 1 , y de salinidad con un refractómetro manual Krüss Optronic HR27-100, con rango de precisión 1.000-1.070 d20, 0-100 ups. El material biológico se preservó en formaldehído al $4 \%$, neutralizado con borato de sodio.

Para estimar la abundancia de las hidromedusas se contabilizaron las muestras totalmente; los valores del volumen de agua filtrada se estandarizaron para $100 \mathrm{~m}^{3}$ (Pavez et al., 2010: 1053). Para la clasificación a nivel específico se siguió a Mayer (1910), Russell (1953), Kramp (1961 y 1968), Ramírez y Zamponi (1981), Segura-Puertas (1984) y Bouillon et al. (2004).

Con las determinaciones de abundancia específica se estableció la estructura de la comunidad, usando los índices de diversidad de Shannon (H') y de equidad de Pielou (J) (Moreno, 2001: 46). Con los valores de riqueza observada se usaron los modelos cuantitativos de acumulación de especies de Clench, Coleman (Cole) y Chao 1, los cuales se han reportado como los más precisos (Walther y Morand, 1998). La riqueza del área analizada se estimó con el software EstimateS 6.0 (Villarreal et al., 2006: 193). Finalmente, el coeficiente de correlación de Spearman $\left(\mathrm{r}_{\mathrm{S}}\right)$ permitió observar la relación entre las variables ambientales determinadas y los indicadores de la estructura de la comunidad.

\section{Resultados}

Los registros más bajos de temperatura se obtuvieron en febrero $\left(18.2-20.2^{\circ} \mathrm{C}\right)$, y los más altos en agosto $\left(27.5-29.0^{\circ} \mathrm{C}\right)$; los valores de salinidad más elevados se encontraron en noviembre (30.7- $38 \%$ ), mientras que los más bajos en octubre (4-32\%) (cuadro 1, figura 2A).

En las muestras de zooplancton se determinaron 22 especies de hidromedusas, pertenecientes a 19 géneros, 14 familias y cinco subclases. En el área estudiada las especies neríticas contribuyeron con la mayor riqueza y densidad, mientras que las oceánicas estuvieron menos representadas tanto en número de especies como en densidad (cuadro 2).

En cuanto a la distribución temporal de las especies, la máxima densidad se observó a finales de la temporada de secas (abril-mayo); la riqueza no tuvo

${ }^{1}$ EstimateS: Statistical Estimation of Species Richness and Shared Species from Samples (versión 6.0) < http://viceroy.eeb.uconn.edu/estimates > [2013, 5 de junio]. 


\section{Cuadro 1. Valores superficiales de temperatura $\left({ }^{\circ} \mathrm{C}\right)$ y salinidad (ups)} de noviembre de 1990 a octubre de 1991

\begin{tabular}{lcccc} 
Meses & \multicolumn{2}{c}{ Temperatura } & \multicolumn{2}{c}{ Salinidad } \\
& Mínima & Máxima & Mínima & Máxima \\
Noviembre & 23.0 & 25.0 & 30.7 & 38.0 \\
Enero & 19.3 & 20.4 & 26.0 & 33.0 \\
Febrero & 18.2 & 20.2 & 23.0 & 32.0 \\
Marzo & 24.0 & 25.0 & 30.0 & 35.0 \\
Abril & 25.0 & 26.0 & 30.0 & 35.0 \\
Mayo & 23.0 & 29.0 & 24.0 & 30.0 \\
Agosto & 27.5 & 29.0 & 18.0 & 30.0 \\
Septiembre & 27.0 & 28.0 & 13.0 & 34.0 \\
Octubre & 24.0 & 26.0 & 4.0 & 32.0 \\
\hline
\end{tabular}

Fuente: Elaboración propia.
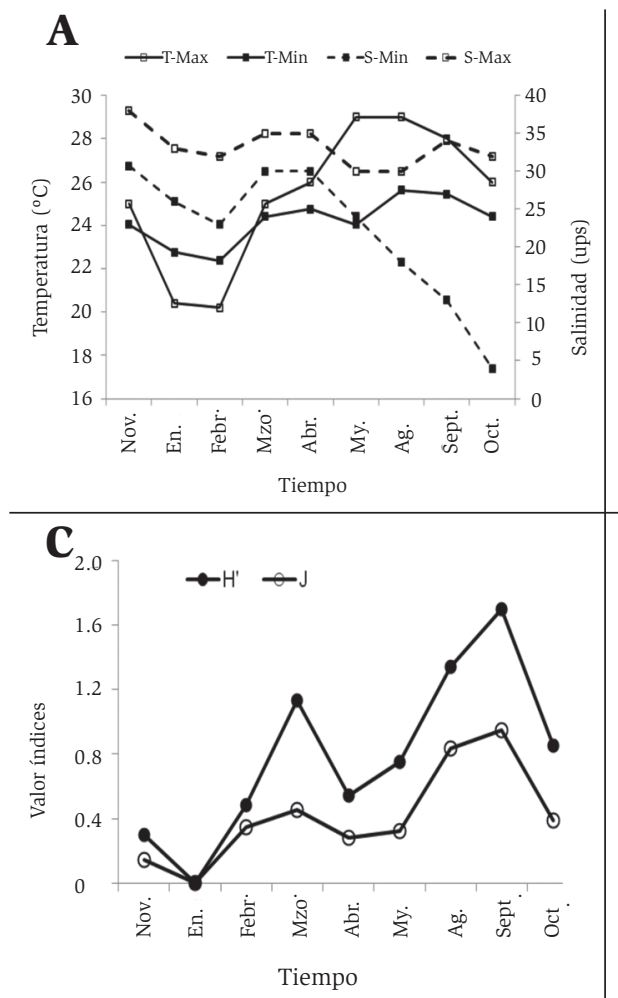
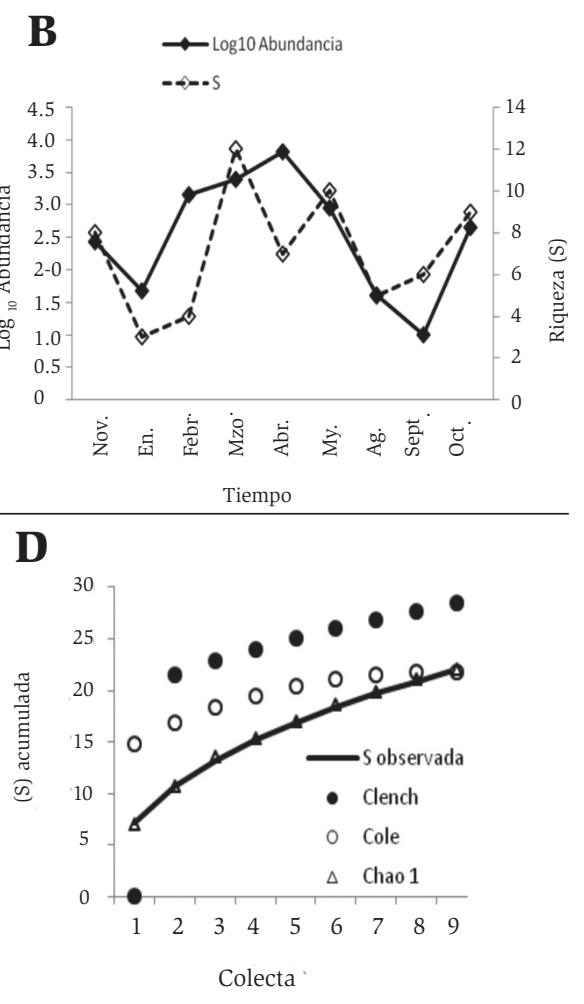

Figura 2.- Indicadores ambientales y de estructura de la comunidad de hidromedusas; $A$ ) temperatura $(\mathrm{T})$ y salinidad (S); $B$ ) Abundancia y riqueza mensual; $C$ ) Índices de diversidad y equidad; $D$ ) Riqueza observada y estimada. Max = máxima; Min = mínima. 
fluctuaciones claras entre temporadas, aunque se presentó una tendencia de mayor riqueza hacia el término de nortes y secas (figura 2B).

Las especies que destacaron por su frecuencia de aparición y abundancia son Obelia sp., Liriope tetraphylla (Chamisso y Eysenhardt, 1821), Persa incolorata McCrady, 1857 y Clytia discoida (Mayer, 1900), que constituyeron el 95.9 $\%$ de la abundancia relativa durante el ciclo analizado (cuadro 2). Obelia sp. fue la especie con abundancia relativa más alta ( $57.8 \%$ del total) y se le observó casi todos los meses -excepto en octubre-, con máxima densidad en secas (abril) y bajas densidades en lluvias. Liriope tetraphylla presentó $27.2 \%$ de abundancia relativa y se registró todos los meses del ciclo analizado, con densidad máxima en nortes (febrero y marzo) y mínima en lluvias, casi desapareciendo en septiembre (cuadro 2).

Persa incolorata fue la tercera especie más abundante (6.0\%), se encontró con mayor densidad en secas; Clytia discoida contribuyó con $4.9 \%$ de la abundancia total, con mayor densidad en secas (abril), se ausentó en lluvias e incrementó en densidad otra vez en octubre (cuadro 2).

La abundancia total tendió a ser más elevada hacia el término de nortes e inicio de secas (febrero-abril), y en este periodo los índices de equidad y diversidad mostraron una tendencia a la baja; mientras que en lluvias la comunidad fue más equitativa (figura $2 \mathrm{C}$ ). La riqueza y abundancia disminuyeron durante lluvias, lo cual coincidió con el aumento en los índices de diversidad y equidad (figuras $2 \mathrm{~B}$ y $2 \mathrm{C}$ ).

La riqueza estimada varió entre 22 (según los modelos Cole y Chao 1) y 28 (con Clench) (figura 2D), de tal forma que la riqueza observada representó del 78.5 al $100 \%$ de la estimada. Una prueba de Kruskal-Wallis mostró que la riqueza observada y la estimada difirieron significativamente $(\mathrm{H}=14.08$; $\mathrm{p}<$ 0.05).

La riqueza observada (S), el índice de equidad (J) y la abundancia mensual no revelaron relación significativa con las variables ambientales analizadas. Solo el índice de diversidad (H') se relacionó de manera significativa con la temperatura mínima $\left(\mathrm{r}_{\mathrm{s}}=0.8 ; \mathrm{p}<0.05\right)$ y la temperatura máxima $\left(\mathrm{r}_{\mathrm{s}}=0.67 ; \mathrm{p}<0.05\right)$. 
Diversidad de las hidromedusas (Cnidaria) de la región nerítica del sureste de Tamaulipas, México

Cuadro 2. Densidad mensual (org/ $100 \mathrm{~m}^{3}$ ) y abundancias de las hidromedusas del área analizada ${ }^{1}$

\begin{tabular}{|c|c|c|c|c|c|c|c|c|c|c|c|c|}
\hline & $\mathbf{N}$ & $\mathbf{E}$ & $\mathbf{F}$ & $\mathbf{M}$ & $\mathbf{A}$ & $\mathbf{M}$ & A & $\mathbf{S}$ & $\mathbf{0}$ & AT & AR & $\mathbf{H}$ \\
\hline \multicolumn{13}{|l|}{$\begin{array}{l}\text { Superclase Hydrozoa } \\
\text { Clase Hydroidomedusa } \\
\text { SubClase Anthomedusae } \\
\text { Familia BougainVillidae }\end{array}$} \\
\hline $\begin{array}{l}\text { Bougainvillia niobe } \\
\text { Mayer, } 1894\end{array}$ & & & & & 21 & 3 & & 1 & 2 & 27 & 0.22 & * \\
\hline $\begin{array}{l}\text { B. platygaster } \\
\text { (Haeckel, 1879) }\end{array}$ & & & & 3 & & & & & & 3 & 0.02 & * \\
\hline $\begin{array}{l}\text { Nemopsis bachei } \\
\text { Agassiz, } 1849\end{array}$ & & & & 2 & & & & & & 2 & 0.02 & * \\
\hline \multicolumn{13}{|l|}{ Familia Cytaeididae } \\
\hline $\begin{array}{l}\text { Cytaeis tetrastyla } \\
\text { Eschscholtz, } 1929\end{array}$ & & & & & & 2 & & & & 2 & 0.02 & * \\
\hline \multicolumn{13}{|l|}{ Familia Corynidae } \\
\hline $\begin{array}{l}\text { Coryne eximia } \\
\text { (Allman, 1859) }\end{array}$ & & & & 43 & 63 & & & & & 106 & 0.88 & * \\
\hline $\begin{array}{l}\text { Slabberia halterata } \\
\text { McCrady, } 1859\end{array}$ & 3 & & & & & & & & & 3 & 0.02 & $*$ \\
\hline \multicolumn{13}{|l|}{ FAMILIA CORYMORPHIDAE } \\
\hline $\begin{array}{l}\text { Euphysora gracilis } \\
\text { (Brooks, 1882) }\end{array}$ & 54 & & & 9 & & 8 & & 1 & 3 & 75 & 0.61 & $*$ \\
\hline \multicolumn{13}{|l|}{ Familia TubulariIdae } \\
\hline $\begin{array}{l}\text { Ectopleura } \\
\text { dumortierii }^{\times} \\
\text {(Van Beneden, 1844) }\end{array}$ & & & & 58 & & & & & 2 & 60 & 0.49 & $*$ \\
\hline \multicolumn{13}{|l|}{$\begin{array}{l}\text { SUbCLASE LEPTOMEDUSAE } \\
\text { FAMILIA EIRENIDAE }\end{array}$} \\
\hline $\begin{array}{l}\text { Eirene pyramidalis } \\
\text { (L. Agassiz, 1862) }\end{array}$ & & & & & & 2 & & & 12 & 14 & 0.11 & $*$ \\
\hline $\begin{array}{l}\text { Eutima gracilis } \\
\text { (Brooks, 1882) }\end{array}$ & & & & & & & & & 19 & 19 & 0.16 & * \\
\hline
\end{tabular}

Simbología: $\times$ nuevo registro para el norte del golfo de México, $\mathrm{H}=$ Hábitat, *nerítica, **oceánica; abundancia total $=$ AT; abundancia relativa $=$ AR

${ }^{1}$ Arreglo taxonómico según Bouillon y Boero (2000). Se presentan las iniciales de los meses analizados. 
Cuadro 2. Densidad mensual (org/ $100 \mathrm{~m}^{3}$ ) y abundancias

de las hidromedusas del área analizada ${ }^{1}$

(continuación)

\begin{tabular}{|c|c|c|c|c|c|c|c|c|c|c|c|c|}
\hline & $\mathbf{N}$ & $\mathbf{E}$ & $\mathbf{F}$ & $\mathbf{M}$ & $\mathbf{A}$ & $\mathbf{M}$ & $\mathbf{A}$ & $\mathbf{S}$ & $\mathbf{0}$ & AT & $\mathbf{A R}$ & $\mathbf{H}$ \\
\hline \multicolumn{13}{|l|}{ FAMiLia LoveneliIdaE } \\
\hline $\begin{array}{l}\text { Eucheilota } \\
\text { duodecimalis } \\
\text { A. Agassiz, } 1862\end{array}$ & 2 & & 21 & 22 & 38 & 9 & 3 & & 17 & 112 & 0.92 & $*$ \\
\hline $\begin{array}{l}\text { E. paradoxica Mayer, } \\
1900\end{array}$ & 3 & & & & & & & & & 3 & 0.02 & * \\
\hline $\begin{array}{l}\text { E. ventricularis }{ }^{\times} \\
\text {McCrady, } 1859\end{array}$ & & & & 14 & & & & & 13 & 27 & 0.22 & * \\
\hline \multicolumn{13}{|l|}{$\begin{array}{l}\text { FAMILIA } \\
\text { CAMPANULARIDAE }\end{array}$} \\
\hline Obelia sp. & 36 & 28 & 169 & 1046 & 5749 & 6 & 11 & 1 & & 7046 & 57.7 & $*$ \\
\hline $\begin{array}{l}\text { Clytia discoida } \\
\text { (Mayer, 1900) }\end{array}$ & & & & 83 & 489 & 7 & 2 & & 19 & 600 & 4.92 & $*$ \\
\hline \multicolumn{13}{|l|}{$\begin{array}{l}\text { SubClase LimNomedusae } \\
\text { FAMILIa Olindidae }\end{array}$} \\
\hline $\begin{array}{l}\text { Gossea brachymera } \\
\text { Bigelow, } 1909\end{array}$ & & & & 2 & & & & & & 2 & 0.02 & $* *$ \\
\hline \multicolumn{13}{|l|}{$\begin{array}{l}\text { SubClase NARComedusa } \\
\text { FAMILIA CuninidaE }\end{array}$} \\
\hline $\begin{array}{l}\text { Cunina octonaria } \\
\text { McCrady, } 1859\end{array}$ & 3 & & & & & & & & & 3 & 0.02 & $* *$ \\
\hline \multicolumn{13}{|l|}{ Familia AEginidae } \\
\hline $\begin{array}{l}\text { Solmundella } \\
\text { bitentaculata (Quoy y } \\
\text { Gaimard, 1833) }\end{array}$ & & & & & & & & 2 & & 2 & 0.02 & $* *$ \\
\hline \multicolumn{13}{|l|}{ FAmilia Solmarisidae } \\
\hline $\begin{array}{l}\text { Solmaris flavescens } \\
\text { (Kölliker, 1853) }\end{array}$ & 31 & & & & & 2 & 7 & 3 & & 43 & 0.35 & $* *$ \\
\hline \multicolumn{13}{|l|}{$\begin{array}{l}\text { SubClASE } \\
\text { TraChyMEDUSAE } \\
\text { FAMILIA GERYONIDAE }\end{array}$} \\
\hline $\begin{array}{l}\text { Liriope tetraphylla } \\
\text { (Chamisso y } \\
\text { Eysenhardt, 1821) }\end{array}$ & 137 & 18 & 1214 & 1155 & 237 & 184 & 19 & 2 & 356 & 3322 & 27.2 & $* *$ \\
\hline
\end{tabular}

Eucheilota

duodecimalis

2

$21 \quad 22$

9 3

E. paradoxica Mayer,

14 
Cuadro 2. Densidad mensual (org/ $100 \mathrm{~m}^{3}$ ) y abundancias de las hidromedusas del área analizada ${ }^{1}$

(finaliza)

\begin{tabular}{|c|c|c|c|c|c|c|c|c|c|c|c|c|}
\hline & $\mathbf{N}$ & $\mathbf{E}$ & $\mathbf{F}$ & $\mathbf{M}$ & $\mathbf{A}$ & $\mathbf{M}$ & $\mathbf{A}$ & S & $\mathbf{0}$ & AT & AR & H \\
\hline \multicolumn{13}{|l|}{$\begin{array}{l}\text { FAMILIA } \\
\text { RHOPALONEMATIDAE }\end{array}$} \\
\hline $\begin{array}{l}\text { Aglaura hemistoma } \\
\text { Perón y Lesueur, } 1810\end{array}$ & & 1 & & & & & & & & 1 & 0.01 & $* *$ \\
\hline $\begin{array}{l}\text { Persa incolorata } \\
\text { McCrady, } 1857\end{array}$ & & & 11 & 13 & 21 & 687 & & & & 732 & 6.01 & $* *$ \\
\hline $\begin{array}{l}\text { Abundancia absoluta } \\
\text { mensual }\end{array}$ & 269 & 47 & 1415 & 2450 & 6618 & 910 & 42 & 10 & 443 & 12204 & & \\
\hline $\begin{array}{l}\text { Abundancia relativa } \\
\text { mensual }\end{array}$ & 2.21 & 0.3 & 11.6 & 20.09 & 54.26 & 7.46 & 0.34 & 0.0 & 3.63 & & 100 & \\
\hline
\end{tabular}

Fuente: Elaboración propia.

\section{Discusión}

Los cambios en la riqueza en comunidades de hidromedusas no son claros entre temporadas, lo cual se ha observado también en comunidades del Caribe mexicano; los cambios estructurales más relevantes en este tipo de comunidades se han reportado en la abundancia, la biomasa y en indicadores como H', el índice de valor de importancia (Ivi) y J (Canché-Canché y Castellanos-Osorio, 2005). En este caso de estudio se encontraron especies dominantes durante nortes y secas, mientras que en época de lluvias la comunidad de hidromedusas neríticas fue más equitativa.

Aunque los cambios estructurales temporales no sean claros (como en este caso), la riqueza de especies es un atributo que describe con claridad la biodiversidad de un ambiente, e incluso puede usarse para comparar localidades diferentes (Magurran, 1988: 23). Por ello, la inferencia de la riqueza por medio de estimadores puede ser muy útil como un indicador de la estructura de las comunidades, sobre todo en aquellos grupos o áreas poco investigados y donde los cambios temporales naturales producen variaciones de dominancia o cuya distribución no es estrictamente aleatoria (Soberón y Llorente, 1993: 480), tal como ocurre con el zooplancton. Sin duda, la diferencia significativa encontrada entre la riqueza observada y estimada se debe a la proyección obtenida con 
la ecuación de Clench, modelo recomendado para aplicarse en sitios de área extensa (Soberón y Llorente, 1993). De esta forma, aunque la riqueza real es similar a la estimada, el resultado obtenido con los estimadores sugiere que la riqueza del área de estudio aumentará en alrededor de $20 \%$ con un incremento en el esfuerzo de muestreo. Este número es acorde con los registros de hidromedusas de la región nerítica del golfo de México (Segura-Puertas, SuárezMorales y Celis, 2003: 3-10).

Los resultados aquí expuestos también soportan la hipótesis de que un mayor esfuerzo de colecta de este tipo de fauna incrementará el número de especies de hidromedusas registradas y conocidas para los océanos del país (Segura-Puertas, Suárez-Morales y Celis, 2003: 11). Por ejemplo, las especies Coryne eximia (Allman, 1859), Slabberia halterata McCrady, 1859, Ectopleura dumortierii Van Beneden, 1844 y E. ventricularis McCrady, 1859 constituyen nuevos registros en el área del golfo de México analizada; estas especies ya habían sido catalogadas en el Pacífico y el Caribe mexicanos (Segura-Puertas, Suárez-Morales y Celis, 2003: 3-10; Segura-Puertas, Celis y Chiaverano, 2009: 374-377).

El número de especies consignadas en esta zona es menor si se comparan con los trabajos de Phillips (1972), Sears (1954: 273), Segura-Puertas, SuárezMorales y Celis (2003: 3-9) y Segura-Puertas, Celis y Chiaverano (2009: 370), pero esto se debe a la amplitud hidrográfica y a las diversas profundidades de los muestreos considerados en estas investigaciones, ya que este estudio se restringe a una franja litoral de la zona costera. La mayoría de las especies registradas aquí ocupan ambientes neríticos (cuadro 2), sin embargo, se reconoce que los ambientes con mayor diversidad de hidromedusas son las regiones epipelágicas 0, incluso, las mesopelágicas (Segura-Puertas, Suárez-Morales y Celis, 2003: 11). Conforme se incrementen los esfuerzos de colecta hacia esas regiones en el área norte del golfo de México puede suponerse, tentativamente, que el número de especies de hidromedusas registradas para el área también se incrementará.

La relación de afluencia y distribución temporal de las especies determinadas en función de las condiciones hidrológicas muestra que el factor vinculado directamente con la diversidad de hidromedusas de la región fue la variación 
de la temperatura del agua, ya que la máxima diversidad se presentó en lluvias, cuando las temperaturas eran las más altas.

En apariencia, la salinidad no presentó ninguna relación con los indicadores de la estructura de la comunidad; empero, tal vez influyó en la distribución temporal de algunas de ellas, ya que las que presentaron mayor abundancia durante el periodo de estudio toleran un rango alto de salinidad, como son Obelia sp., L. tetraphylla, C. discoida o E. duodecimalis A. Agassiz, 1862, consideradas eurihalinas o euritermas. Asimismo, en el área trabajada se tiene la influencia directa de aguas continentales provenientes del río Pánuco, lo cual genera variaciones frecuentes en la salinidad.

Las especies de Obelia se consideran neríticas, cosmopolitas y abundantes en las zonas litorales; en este estudio Obelia sp. registró en secas su máxima abundancia, y en este tiempo se encontró también que la mayoría de los organismos de esta especie registrados eran jóvenes. Por su parte, Liriope tetraphylla es una especie oceánica eurihalina y presenta una amplia distribución en los océanos de las zonas templadas y cálidas (Alvariño, 1968: 67; Segura-Puertas, 1992: 355-356; Pavez et al., 2010: 1051), en este análisis, su máxima abundancia se verificó en nortes, también con una mayoría de organismos jóvenes; aparentemente en esta época la temperatura es óptima para la reproducción de esta especie, tal como lo mencionó Canudas (1979: 186), quien reportó el invierno como la mejor época para la producción de yemas de L. tetraphylla en la laguna de Términos, también en el golfo de México.

Persa incolorata McCrady, 1857 es una especie oceánica con una amplia distribución en las zonas templadas y cálidas de los océanos Atlántico y Mediterráneo, y fue la tercera especie más abundante en este estudio: su máxima abundancia ocurrió en secas, época en que la mayoría de los organismos registrados eran jóvenes. La comunidad presentó mayor equidad durante lluvias debido a que las tres especies dominantes mencionadas estuvieron casi ausentes durante esta temporada.

Las especies registradas indican un aporte faunístico causado por la acción de diversas corrientes en el área estudiada; este proceso contribuye a que poblaciones de diferentes especies avancen y, si las condiciones les son favorables, en muchos casos logren establecerse, produciendo enriquecimiento faunístico 164 a escala regional, tal como lo mencionó Segura-Puertas (1991). 


\section{Agradecimientos}

La doctora Lourdes Segura Puertas (†) corroboró la identificación a nivel específico de los organismos. La lectura previa de María del Rosario Martínez Hernández e Inocente López Salgado contribuyó a mejorar el escrito. Agradecemos a la Universidad de Quintana Roo por proporcionar el tiempo y el espacio para concluir el presente trabajo a través del apoyo al Cuerpo Académico Vulnerabilidad y Biodiversidad de Sistemas Acuáticos Continentales y Costeros (uoroo-Unidad Cozumel).

\section{Fuentes consultadas}

Alvariño, A. (1968). "Los quetognatos, sifonóforos y medusas en la región del Atlántico ecuatorial bajo la influencia del Amazonas". Anales del Instituto de Biología (serie Ciencias del Mar y Limnología), 1, 41-76. (1972). "Zooplancton del Caribe, Golfo de México y regiones adyacentes del Pacífico”, en Memorias del IV Congreso Nacional de Oceanografía. México: Universidad Nacional Autónoma de México, 223-247.

Arriaga-Cabrera, L. et al. (coords.) (1998). Regiones marinas prioritarias de México. México: Comisión Nacional para el Conocimiento y Uso de la Biodiversidad.

Bouillon, J. y F. Boero (2000). Thalassia Salentina, 24, Phylogeny and Classification of Hydroidomedusae, $291 \mathrm{pp}$.

et al. (2004). Scientia Marina, 68 (suppl. 2), Fauna of the Mediterranean Hydrozoa, $454 \mathrm{pp}$.

Canché-Canché, V. E. e I. Castellanos-Osorio (2005). “Medusas (Cnidaria) de la Bahía de la Ascensión, Quintana Roo, México (1997)”. Hidrobiológica, 15 (1), 65-72.

Canudas, A. (1979). "Contribución al conocimiento de las medusas (Coelenterata) de la Laguna de Términos, Campeche, México". Anales del Centro de Ciencias del Mar y Limnología, 6 (1), 183-188.

Correia-Valencia, K. (1992). "Medusas del Golfo de México y litoral norte/noreste de Brasil (ocurrencia y distribución)”. Tesis doctoral. México: Facultad de Ciencias-Universidad Nacional Autónoma de México, 284 pp. Gómez-Aguirre, S. (1991). “Contribución al estudio faunístico de celenterados y 
ctenóforos del plancton estuarino del noroeste de México". Anales del Instituto de Biología (serie Zoología), 62 (1), 1-10.

Kramp, P. (1961). Journal of the Marine Biological Association of the United Kingdom, 40, Synopsis of the Medusae of the World, $469 \mathrm{pp}$. (1968). The Hydromedusae of the Pacific and Indian Ocean. Sections II and III (Dana Report 72), Copenhague: The Carlsberg Foundation, 200 pp.

Magurran, A. E. (1988). Ecological Diversity and Its Measurement. Nueva Jersey: Princeton University Press, $179 \mathrm{pp}$.

Mayer, A. G. (1910). Medusae of the World, vols. I, II y III. Washington: Carnegie Foundation, $735 \mathrm{pp}$.

Moreno, C. E. (2001). Métodos para medir la biodiversidad. Zaragoza: Programa Iberoamericano de Ciencia y Tecnología para el Desarrollo/Oficina Regional de Ciencia y Tecnología para América Latina y el Caribe-Unesco/Sociedad Entomológica Aragonesa (SEA) (Manuales y Tesis SEA, 1), $84 \mathrm{pp}$.

Ocaña-Luna, A., G. Hernández-Batún y M. Sánchez-Ramírez (2008). “Abundancia y distribución de juveniles de Farfantepenaeus aztecus (Ives 1891), F. duorarum (Burkenroad 1939) y Litopenaeus setiferus (Linnaeus, 1767) en la Laguna Madre, Tamaulipas, México”. Hidrobiológica, 18 (3), 199-208.

Pavez, M. et al. (2010). "Distribution of Carnivorous Gelatinous Zooplankton in the Upwelling Zone off Central Chile (Austral Spring 2001)”. Journal of Plankton Research, 32 (7), 1051-1065.

Phillips, P. J. (1972). "The Pelagic Cnidaria of the Gulf of Mexico”. Tesis doctoral. San Antonio: Universidad de Texas, $211 \mathrm{pp.}$

Ramírez, F. C. y M. O. Zamponi (1981). "Hydromedusae”, en D. Boltovskoy (ed.). Atlas del zooplancton del Atlántico sudoccidental y métodos de trabajo con el zooplancton marino. Mar del Plata: Instituto Nacional de Investigación y Desarrollo Pesquero, 443-469.

Raskoff, K. A. (2001). “The Impact of El Niño Events on Populations of Mesopelagic Hydromedusae”. Hydrobiologia, 451, 121-129.

Russell, F. (1953). The Medusae of the British Isles: Anthomedusae, Leptomedusae, Limnomedusae, Trachymedusae and Narcomedusae medusa. Cambridge: Cambridge University Press, 530 pp. 
Sears, M. (1954). "Hydromedusae of the Gulf of México”, en P. S Galtsoff (ed.). Gulf of Mexico, Its Origin, Waters, and Marine Life. Washington: United States Fish and Wildlife Service, 273-274.

Segura-Puertas, L. (1984). "Morfología, sistemática y zoogeografía de las medusas (Cnidaria: Hydrozoa y Scyphozoa) del Pacífico tropical oriental”. Tesis doctoral. México: Facultad de Ciencias-Instituto de Ciencias del Mar y Limnología-unam, 320 pp.

(1991). "Medusas (Cnidaria: Hydrozoa y Scyphozoa) de la región del Domo de Costa Rica”. Revista de Biología Tropical, 39 (1), 159-163. (1992). "Medusae (Cnidaria) from the Yucatan Shelf and Mexican Caribbean”. Bulletin of Marine Science, 51 (3), 353-359.

-------, L. Celis y L. Chiaverano (2009). "Meduzoans (Cnidaria: Cubozoa, Scyphozoa and Hydrozoa) of the Gulf of Mexico”, en D. L. Felder y D. K. Camp. Gulf of Mexico Origin, Waters, and Biota. vol. 1. Biodiversity. Texas: Texas A\&M University Press, 369-379.

--------, E. Suárez-Morales y L. Celis (2003). "A Checklist of the Medusae (Hydrozoa, Scyphozoa and Cubozoa) of Mexico”. Zootaxa, 194, 1-15.

Signoret, M. (1969). "Contribución al conocimiento de las medusas de las lagunas de Tamiahua y Alvarado, Veracruz”. Tesis. México: Facultad de Ciencias-Universidad Nacional Autónoma de México, 52 pp.

Soberón, J. y J. Llorente (1993). “The Use of Species Accumulation Functions for the Prediction of Species Richness”. Conservation Biology, 7 (3), 480-488.

Suárez- Morales, E., L. Segura-Puertas y R. Gasca (1995). “Medusas (Cnidaria: Hydrozoa) de la Bahía de Chetumal, Quintana Roo, México (19901991)". Caribbean Journal of Science, 31 (3-4), 243- 251.

Villarreal, H. et al. (2006). Manual de métodos para el desarrollo de inventarios de biodiversidad. Bogotá: Instituto de Investigación de Recursos Biológicos Alexander von Humboldt, $236 \mathrm{pp}$.

Walther, B. A. y S. Morand (1998). "Comparative Performance of Species Richness Estimation Methods”. Parasitology, 116 (4), 395-405. 